\title{
Levantamento do seio maxilar pela técnica da janela lateral uma revisão da
}

\section{literatura}

\author{
Lifting the maxillary sinus using the side window technique a literature review \\ Levantamiento del seno maxilar mediante la técnica de ventana lateral una revisión de la literatura
}

Recebido: 18/08/2021 | Revisado: 26/08/2021 | Aceito: 27/08/2021 | Publicado: 29/08/2021

Flávia Caroline Teixeira de Sousa

ORCID: https://orcid.org/0000-0001-7499-1781 Faculdade Patos de Minas, Brasil

E-mail: flaviatxsousa@outlook.com

Marcelo Dias Moreira de Assis Costa

ORCID: https://orcid.org/0000-0001-9148-3674

Universidade Federal de Uberlândia, Brasil

E-mail: marcelodmac@yahoo.com.br

Lia Dietrich

ORCID: https://orcid.org/0000-0001-7887-8591 Universidade Federal dos Vales do Jequitinhonha e Mucuri, Brasil

E-mail: lia.dietrich@ufvjm.edu.br

\begin{abstract}
Resumo
Nas últimas décadas, a implantodontia vem sendo bastante utilizada na reabilitação de áreas edêntulas. Uma das condições essenciais para a colocação dos implantes é a altura óssea suficiente. Quando tal condição não é satisfeita, propõe-se o procedimento de levantamento ou elevação do seio maxilar. Essa elevação pode ser obtida pela utilização da abordagem da janela lateral ou "elevação direta do seio", que é uma técnica cirúrgica traumática que permite o aumento da quantidade de osso do maxilar posterior. Diante disso, esta pesquisa buscar conhecer a técnica da janela lateral, visando esclarecer seus benefícios, indicações e limitações. Além disso, apresenta uma revisão bibliográfica pautada na literatura e em artigos publicados em plataformas como Pub Med, Scielo e Google Acadêmico nos últimos 10 anos. Foi possível verificar que, dentre a várias técnicas cirúrgicas existentes para o levantamento do seio maxilar, a abordagem da janela lateral tem se mostrado a melhor, dada a sua capacidade de enxertia óssea atingir elevadas alturas. Altas taxas de sucesso dependem de um adequado diagnóstico, manejo clínico, cirúrgico e de profissionais capacitados.
\end{abstract}

Palavras-chave: Implantodontia; Elevação do seio maxilar; Abordagem da janela lateral.

\begin{abstract}
In recent decades, implantology has been widely used in the rehabilitation of endentulous areas. One of the essential conditions for implant placement is sufficient bone height. When this condition is not met, the procedure for lifting or elevating the maxillary sinus is proposed. This elevation can be achieved by using the side window approach or "direct sinus elevation", which is a traumatic surgical technique that allows an increase in the amount of bone in the posterior jaw.Therefore, this research seeks to know the technique of the side window, aiming to clarify its benefits, indications and limitations. In addition, it presents a bibliographic review based on the literature and articles published on platforms such as Pub Med, Scielo and Google Scholar in the last 10 years. It was possible to verify that, among the several existing surgical techniques for the lifting of the maxillary sinus, the lateral window approach has been shown to be the best, given its bone grafting capacity to reach high heights. High success rates depend on an adequate diagnosis, clinical, surgical management and trained professionals.
\end{abstract}

Keywords: Implantology, Maxillary sinus elevation; Side window approach.

\section{Resumen}

En las últimas décadas, la implantología ha sido ampliamente utilizada en la rehabilitación de áreas endentadas. Una de las condiciones esenciales para la colocación de implantes es una altura ósea suficiente. Cuando no se cumple esta condición, se propone el procedimiento de elevación o elevación del seno maxilar. Esta elevación se puede lograr mediante el abordaje de ventana lateral o "elevación directa del seno", que es una técnica quirúrgica traumática que permite aumentar la cantidad de hueso en la mandíbula posterior. Así, esta investigación busca conocer la técnica de la ventana lateral, con el objetivo de esclarecer sus beneficios, indicaciones y limitaciones. Además, presenta una revisión bibliográfica basada en la literatura y artículos publicados en plataformas como Pub Med, Scielo y Google Scholar en los últimos 10 años. Se pudo comprobar que, entre las diversas técnicas quirúrgicas existentes para la elevación del seno maxilar, el abordaje de la ventana lateral ha demostrado ser el mejor, dada su capacidad de injerto 
óseo para alcanzar alturas elevadas. Las altas tasas de éxito dependen de un adecuado diagnóstico, manejo clínico, quirúrgico y profesionales capacitados.

Palabras clave: Implantología; Elevación del seno maxilar; Enfoque de ventana lateral.

\section{Introdução}

As reabilitações de áreas edêntulas utilizando implantes dentários tem sido bastante empregada nas últimas décadas. Devido a evolução dos implantes, componentes e biometrias a implantodontia tornou-se, assim, uma alternativa de tratamento inovadora, real e viável para a reabilitação oral (Moraes; Tomasi \& Jonas, 2018); David et al., 2018).

A altura óssea suficiente para colocação dos implantes é uma condição essencial para a reabilitação. Adicionalmente, para garantir o sucesso e a longevidade dos implantes, é fundamental que exista quantidades mínimas de tecido ósseo remanescente, com espessuras e comprimentos adequados para suportar os esforços mastigatórios (Correia, 2020; Batista et al., 2020).

Quando tais condições não são satisfeitas, propõe-se o procedimento de levantamento ou elevação do seio maxilar, que tem por função aumentar a altura de osso residual na maxila posterior através da elevação da membrana de Schneider (Batista et al., 2020).

O Sinus Lift, também denominada de elevação do seio maxilar, é uma cirurgia que permite o aumento da quantidade de osso do maxilar posterior. Geralmente, utilizada para colocar implantes dentários posteriormente, quando há perda de um dente natural e consequente perda de volume ósseo na zona desdentada (Bacelar \& Guimarães Neto, 2019).

A região posterior da maxila é uma região desafiadora para odontologia quando comparadas com outras áreas da boca. Isso ocorre, em função da qualidade óssea menos favorável e da presença do seio maxilar que limita muitas regiões específicas da maxila. O seio maxilar é uma cavidade pneumatizada, com formato piramidal, frequentemente reforçada por septos intra sinusais (Poli, 2017).

Uma alternativa é a utilização da abordagem da janela lateral ou "elevação direta do seio", que é uma técnica cirurgia traumática, inicialmente desenvolvida por Cadwell-Luc e que permite o aumento da quantidade de osso do maxilar posterior. Esta técnica consiste em realizar uma janela óssea na parede medial do seio maxilar. Posteriormente, reposiciona-se a membrana de Schneider numa posição superior e preenche-se a nova área formada com um material de enxerto. Atualmente, é uma técnica amplamente usada, considerada previsível e viável (Correia et al., 2012; Bacelae \& Guimarães Neto, 2019).

Diante do exposto, esse trabalho tem como objetivo a realização de uma revisão bibliográfica sobre a elevação do seio maxilar através da técnica da janela lateral. Apresentando a relevância da abordagem, suas indicações, contraindicações e aplicabilidade.

\section{Metodologia}

Para o conhecimento do método do levantamento do seio maxilar, pela abordagem da técnica da janela lateral, visando esclarecer seus benefícios, indicações e limitações. Realizou-se uma busca sistemática trabalhos científicos no banco de dados do Pub Med, Scielo e Google Acadêmico, que formam publicados nos últimos 10 anos. A busca foi realizada com as palavras-chave: "sinus lift", "Elevação do seio maxilar", "técnicas de elevação do seio maxilar', "abordagem da janela lateral" e "side window technique". Os artigos deveriam ser meta-analises, revisões sistemáticas, ensaios clínicos randomizados ou de controlo e revisões.

Os critérios de seleção foram trabalhos científicos (artigos, monografias) publicados entre 2011 e 2021, nos idiomas de português e inglês, que retratassem o tema discutido. Ao todo foram utilizados 24 trabalhos considerados relevantes para a pesquisa. 


\section{Referencial Teórico}

Com o avanço da idade do paciente e a perca dos dentes, surge a necessidade do equilíbrio entre o sistema funcional, estético e fonético. Para solucionar esse problema, a utilização dos implantes dentários para reabilitação vem sendo bastante empregada desde 1980, devido a evolução do procedimento, instrumentos e insumo (David et al., 2018).

As áreas edêntulas apresentam como consequências, além da reabsorção óssea do osso alveolar, a pneumatização dos seios maxilares, o que muitas vezes inviabiliza a instalação dos implantes. O levantamento de seio maxilar é indicado para pacientes com reabsorção óssea que necessitam reabilitação (Albuquerque et al., 2014).

A quantidade adequada de altura óssea é uma condição fundamental para a reabilitação com implantes dentários. À vista disso, em alguns casos, é necessário o procedimento de levantamento do seio maxilar, que tem por finalidade aumentar a altura de osso residual na maxila posterior através da elevação da membrana de Schneider (Batista et al., 2020).

O levantamento do seio maxilar, também conhecida por sinus lift, é um procedimento cirúrgico que possui o objetivo de reabilitar áreas edêntulas do maxilar posterior com reabsorções ósseas. Atualmente, é uma técnica bem aceita, documentada, relativamente simples e previsível (Raja, 2009).

Conforme descrito por Batistis, Júnior e Wichnieski (2011), o seio maxilar, maior dos seios paranasais, é uma cavidade pneumática contida no interior da maxila, osso constituinte do terço médio da face. Possui formato piramidal e é frequentemente reforçada por septos intra sinusais. A sua dimensão varia de indivíduo para indivíduo, em um adulto, em média, apresenta $35 \mathrm{~mm}$ de base e $25 \mathrm{~mm}$ de altura (Raja, 2009).

O seio maxilar é delimitado por uma membrana muita fina, membrana Schneideriana, revestida por uma camada de epitélio pseudoestratificado ciliado e possui um tecido conjuntivo altamente vascularizado, a membrana está aderida ao osso subjacente (Lin et al., 2016; Poli, 2017). O epitélio ciliado permite a passagem de fluidos em direção ao meato nasal. As estruturas abaixo do seio consistem na crista alveolar e nos dentes posteriores da maxila (Raja, 2009).

Métodos específicos foram desenvolvidas para a região posterior do maxilar e são necessários para a instalação de implantes quando não há altura óssea suficiente (Misch, 2009). A reabilitação com os implantes dentários demonstraram ser um método eficaz de reposição de peças na região posterior do maxilar, entretanto, apresentam um grande desafio, pois o sucesso do implante está diretamente relacionado com a quantidade e a qualidade óssea (Correia et al., 2012; Misch, 2009).

Segundo Correia (2020), a técnica cirúrgica de elevação do seio maxilar apresenta o maior risco de infecção quando comparados a outros tipos de procedimentos cirúrgicos, devendo ser seguidos uma lista de cuidados à risca para que se evite a contaminação.

A escolha da técnica a ser empregada, é pautada na estrutura óssea residual remanescente e no ganho de altura óssea necessária, esse procedimento pode ser realizado de modo traumático ou atraumático. De forma que o espaço formado abaixo da membrana Schneideriana é enxertada e/ou implementado, utilizando biomaterial, osso autógeno, osso halógeno ou a combinação desses (Batista et al., 2020; David et al., 2018).

O procedimento cirúrgico traumático, como a técnica de janela lateral, é mais invasivo do que a método atraumático. No primeiro método o acesso ao assoalho do seio é através de uma incisão na crista do rebordo alveolar, rebatendo o tecido mucoso com a finalidade de expor o tecido ósseo, aumentando a visualização do campo operatório. Por outro lado, a cirurgia atraumática, o osso não é removido, no método o osso próximo à cortical da cavidade sinusal é empurrado para cavidade apical, com auxílio de osteótomos de extremidade côncava. Nesta técnica não haverá contato direto do instrumental e a membrana do seio maxilar (Reis \& Calixto, 2013).

Diante do exposto, será discorrido e discutido a elevação do seio maxilar pela utilização da técnica da janela lateral (traumática).

A técnica de janela lateral foi inicialmente desenvolvida por Cadwell-Luc e em 1975 modificada por Tatun, 
reportando a entrada no seio maxilar (Raja, 2009; Correia et al., 2012). O método cirúrgico consiste na abertura de uma janela óssea na parede lateral do seio maxilar com instrumentos rotativos ou piezoeléctricos. Posteriormente, eleva-se a membrana de Schneider e preenche-se a área formada com material de enxerto (Correia et al., 2012; Correia, 2020).

A aplicação da técnica da janela lateral é indicada nas seguintes situações (Correia et al., 2012; Correia, 2020; Bacelar \& Guimarães Neto,2019):

1. Quando a altura óssea residual não possibilita a colocação de implantes com comprimento standard ou a utilização de técnicas de elevação menores.

2. Quando a colocação do implante ocorre numa única etapa, realiza-se a elevação do seio simultaneamente a colocação dos implantes, a altura óssea mínima deve ser de $5 \mathrm{~mm}$;

3. Quando se realiza a elevação do seio e posteriormente a colocação de implantes, procedimento em duas etapas, a altura óssea residual varia entre 1 a $4 \mathrm{~mm}$;

4. Quando e necessário inserir uma elevada quantidade de biomaterial, uma vez que, só e possível através deste tipo de técnica.

5. Quando existem inúmeros septos em causa.

Segundo exposto pelos autores Esposito et al. (2010) essa técnica apresenta algumas contraindicações antes da sua aplicação como:

1. Sinusite aguda ou crônica não tratadas.

2. Patologias nos seios maxilares como tumores ou quistos.

3. Problemas psicológicos.

4. Fumantes excessivos.

As técnicas de levantamento do seio maxilar são previsíveis e apresentam sucesso a longo prazo, entretanto, complicações e falhas também são relatas após as cirurgias de implante (Salmen et al., 2017).

A perfurações da membrana de Scheneider, no momento da separação ou da realização da janela óssea, é uma das complicações mais comum (10\% a 34\%). Ao perfurar a membrana podemos deparar-nos com problemas como: infecção sinusal aguda ou crônica, edema, sangramento, deiscência da ferida, perda do material de enxerto ósseo e interrupção da função fisiológica normal dos seios da face (Gandhi,2017; Correia et al., 2012; Bacelar \& Guimarães Neto,2019).

Essas intercorrências podem ser evitadas levando-se em conta fatores importantes como a identificação da quantidade e qualidade óssea do paciente, os princípios fisiológicos da cicatrização dos tecidos e o conhecimento pleno da técnica cirúrgica pelo cirurgião-dentista. Recentemente, diversos estudos tem mostrado bons resultados quando a elevação do seio maxilar é feita pela técnica da janela lateral (Batista et al., 2020).

O pesquisador Guerrero (2015) relatou em sua pesquisa as complicações e os resultados de implantes dentários realizados simultaneamente ou em estágios, que foram colocados em 101 procedimentos de aumento de seio nasal através da abordagem de janela lateral. Ao todo 68 pacientes passaram pelo procedimento de aumento dos seios da face, foram instalados 114 implantes, sendo 65 em simultaneamente e 76 em duas fases. $\mathrm{O}$ autor concluiu que a técnica possuiu alta taxa de sucesso. Entretanto, algumas complicações podem afetar o tratamento clínico, além disso, a colocação simultânea de implantes afeta significativamente sua chance de sucesso.

Kim et al. (2020) compararam em seu estudo a taxa de sobrevivência de implantes que foram colocados utilizando a 
técnica da janela lateral em 1 ou 2 estágios, realizados em osso residual inferior a $4 \mathrm{~mm}$. A pesquisa foi desenvolvida baseada em prontuários odontológicos e radiografias obtidas de pacientes que foram submetidos a técnica da janela lateral de março de 2006 a junho de 2014, posteriormente divididos em grupos de 1 e 2 estágios de acordo com o procedimento realizado. A pesquisa demonstrou que nenhuma diferença significativa foi encontrada na taxa de sobrevivência acumulativa de 10 anos dos implantes entre os grupos de estágio 1 e 2.

Lin et al. (2016) investigaram em seu estudo através de imagens de tomografia computadorizada de feixe cônico (TCFC) a espessura da membrana sinusal de pacientes que foram submetidos a técnica da janela lateral. Adicionalmente, os autores analisaram a influência que a espessura da membrana Schneideriana tem sobre sua perfuração durante a mesma técnica. Entre os anos de 2010 e 2013, foram selecionados 73 pacientes com 81 procedimentos de elevação do seio de face. Cada indivíduo possuía imagens de TCFC do início e imediatamente após a cirurgia. Avaliou-se, os valores e a correlação entre as espessura e morfologia da membrana, taxa de perfuração, altura óssea residual e elevada. O estudo demostrou que a espessura da membrana possui correlação estatística significativa com a perfuração. Além disso, a taxa de perfuração foi mais baixa quando a espessura da membrana era de $5 \mathrm{~mm}$.

Os pesquisadores Lee; Lin e Morton (2013) relataram as complicações encontradas no decorrer de 100 procedimento consecutivos de elevação do seio maxilar, através da técnica da janela lateral. Foram avaliados 86 pacientes, 42 homens e 44 mulheres, que necessitaram de 100 procedimento consecutivos entre março de 2008 e fevereiro de 2011.Registrou-se as alturas ósseas residuais do pré-tratamento e a presença de septos, adicionalmente, foram relatadas as incidências de complicações sinusais intra ou pós-operatórias. Conforme os resultados, a elevação do assoalho do seio nasal, pela técnica da janela lateral, se mostrou uma abordagem previsível para gerenciar a deficiência de volume ósseo na maxila posterior, entretanto pode apresentar complicações.

Os autores Erdem, Çiftçi e Acar (2016) fizeram o acompanhamento clínico e radiográfico de implantes colocados em seio maxilar pela técnica da janela lateral, com alturas ósseas alveolares residuais mínimas ( $\leq 3 \mathrm{~mm}$ ). O estudo ocorreu em um período de 3 anos, um total de 28 assoalhos dos seios da face foram aumentados com xenoenxerto e 58 implantes foram colocados. Pode-se concluir que a taxa de sucesso dos implantes colocados com a abordagem em estágios nos seios maxilares, que possuíam altura do osso alveolar residual de $\leq 3 \mathrm{~mm}$, foi alta no intervalo de 3 anos.

Atualmente, é uma técnica amplamente empregada, confiável e que permite a colocação de implantes e reabilitação protética da maxila posterior edêntula atrófica. Este fato indica que esta abordagem é um procedimento confiável que permite a colocação de implantes e reabilitação protética da maxila posterior edêntula atrófica (Bacelar \& Guimareias Nero, 2019; Lin et al., 2016).

\section{Discussão}

A técnica da janela lateral para elevação dos seios da face é considera um procedimento previsível, bem documentado e com baixa morbidade. No entanto, não é isento de complicações, que podem acarretar no aumento do tempo, no custo total do tratamento, no insucesso dos implantes, em cirurgias adicionais e em questões médico-legais. Devido a isso, os cirurgiões devem reconhecer os fatores de risco para prever complicações (Tükel \& Tatli, 2018).

Mazaro e colaboradores (2013) ressaltaram que a técnica da janela lateral, apesar de ser mais invasiva, é indicada quando há uma extensa pneumatização do seio maxilar e a necessidade de um grande aumento ósseo. Além disso, segundo Correia (2020), é uma técnica cirúrgica recomendada quando a altura óssea residual não permite a colocação de implantes de comprimento standard ou o uso de técnicas de elevação menores (ex. técnica dos osteótomos).

A abordagem da janela lateral possibilita aumentar a altura óssea vertical em mais de $9 \mathrm{~mm}$, enquanto que a abordagem de osteótomo pode aumentar essa altura de 3 para $9 \mathrm{~mm}$. Embora, os procedimentos de osteótomo e janela lateral 
possam ajudar os médicos a superar os desafios de colocação de implantes, a altura óssea residual pré-implante é de suma importância para determinar a sobrevivência desses implantes (Al-Dajani, 2016).

Batistas et al. (2020) puderam concluir que o levantamento do seio maxilar, pela técnica da janela lateral, associado à instalação imediata de implantes osseointegrados é uma opção terapêutica eficaz, bem documentada e altamente previsível para a reabilitação da maxila posterior atrófica.

Morares, Tomas e Jonas (2018) realizaram uma revisão na literatura para a averiguação da técnica de levantamento do seio maxilar com enxerto de osso autógeno. Os autores concluíram que dentre as várias técnicas disponíveis para o levantamento do seio maxilar, a abordagem da janela lateral se apresentou como a primeira escolha para os cirurgiões dentista, por manifestar características e resultados previsíveis para o procedimento cirúrgico em regiões posteriores de maxila.

Os pesquisadores Lin et al., 2016 relataram que a complicação mais ocorrida durante o aumento do seio pela técnica da janela lateral foi a perfuração da membrana sinusal. A perfuração da membrada tem um impacto importante na integridade dos seios da face e pode comprometer a sobrevivência do enxerto ósseo (Al-Dajani, 2016).

Lee, Lin e Morton (2013) descreveram que, além da ruptura da membrana sinusal, pode ocorrer também infecção, deiscência da ferida, perda do enxerto e falha do implante. Segundo os autores é imprescindível que o clínico entenda como reconhecer e resolver essas complicações.

A ocorrência da perfuração da membrana sinusal pode estar relacionada a fatores locais, cúpulas radiculares, presença de septos ósseos, além de patologias sinusais prévias como o tabagismo. A perfuração dessa membrana não é uma contra indicação absoluta para que se possa prosseguir com o tratamento, uma vez que, perfurações menores que $10 \mathrm{~mm}$ de extensão podem ser tratadas e reparadas (Albuquerque et al., 2014).

Bacelar e Guimaraes Neto (2019) reafirmaram a necessidade do profundo conhecimento da anatomia do maxilar devido aos riscos da perfuração da membrana de Scheneider, que pode desencadear excessiva hemorragia, lesão do feixe neurovascular infraorbitário, migração do implante; edema, sensibilidade dos dentes adjacentes, infecção do enxerto, sinusite, quistos e as demais complicações já citadas anteriormente. Todavia, a abordagem da janela lateral pode ser realizada em uma ou duas fases cirúrgica. Caso for realizada em uma única fase, a altura óssea residual tem que estar entre os 4 e os $5 \mathrm{~mm}$. Em duas fases, terá que existir uma altura de $4 \mathrm{~mm}$ ou menos.

Quando realizado em uma única fase cirúrgica, a elevação de seio maxilar ocorre simultaneamente com a colocação do implante. Esse método apresenta as vantagens de diminuir o tempo cirúrgico, o custo e a morbidade. Em vista de que não é necessário fazer uma segunda fase cirúrgica, entretanto, é necessário que a qualidade e a quantidade de ossos sejam suficientes para que exista estabilidade primária. Por outro lado, em duas fases, realiza-se primeiro a elevação da membrana e a colocação do material de enxerto adequado, somente depois que se coloca o implante. À vista disso, são necessários dois tempos de espera: um para a maturação e consolidação do enxerto, variável entre os 6 e os 9 meses, e outro para a osseointegração dos implantes. A técnica apresenta a vantagem de diminuir a probabilidade de infecção do enxerto ósseo e permite ao profissional uma avaliação da colocação do implante em uma região mais adequada (Moniz, 2020).

Por outro lado, Guerrero (2015) demonstrou em seu estudo que o aumento do seio usando a abordagem da janela lateral está associada a uma baixa incidência de complicações graves e a uma alta taxa de sobrevivência de implante. Evidenciando que a abordagem é um procedimento confiável, entretanto, há maiores riscos de falha quando a colocação do implante é realizada pela janela lateral de um estágio.

Quando o sucesso da técnica é medido pelo resultado do paciente (sucesso do procedimento de enxerto), a alta taxa de sucesso atingida é devido que as complicações são mínimas e podem ser minimizadas pela seleção adequada do uso de antibiótico preventivo, pela boa técnica cirúrgica e pelo tratamento das complicações intra-operatórias e pós-operatórias. Já as taxas de sobrevives dos implantes obtidas foram de 96-98\%, tais resultados são consequências da tomada de decisão 
apropriada em relação a superfície do implante, do material do enxerto e da colocação de uma membrana de barreira sobra a janela (Testori et al., 2020).

Ghandi (2017), conclui em seu trabalho que o método da janela lateral oferece uma melhor visualização da membrana sinusal. Para profissionais inexperientes, a opção da piezocirurgia é melhor do que outros dispositivos para a preparação da janela lateral. Adicionalmente, para a técnica da janela lateral ou elevadores indiretos, a altura da elevação deve ser apenas o suficiente para acomodar o implante de comprimento adequado.

Em sua pesquisa Diniz et al. (2012), destacam que o sucesso do método do levantamento do seio maxilar depende de fatores como a capacidade proliferativa da área receptora, do material enxertado e das atividades metabólicas do organismo.

Os resultados bem sucedido da técnica dependem do diagnóstico, do manejo clínico, cirúrgico e protético adequado. Além disso, alguns outros fatores também devem ser levados em conta como a quantidade de remanescente ósseo, a estabilidade primária do implante no momento da instalação, a manutenção da integridade da membrana sinusal e a saúde do seio maxilar prévio (Testori, 2020; Poli, 2017).

\section{Conclusão}

Diante do exposto, pode-se afirmar que a técnica da janela lateral é um procedimento seguro, eficaz, bem documentado e previsível. Dentre as diversas técnicas disponíveis, essa tem se mostrado a melhor devido a sua capacidade de enxertia óssea atingir elevadas alturas, quando sua utilização é possível.

Os altos índices de sucesso dependem de um adequado diagnóstico, manejo clínico, e cirúrgico, além de contar com profissionais capacitados. Complementarmente, deve-se seguir as corretas indicações e contraindicações da abordagem e avaliar em quantas fases, uma ou duas, o procedimento precisará ser realizado.

\section{Referências}

Al-Dajani, M. (2016). Recent trends in sinus lift surgery and their clinical implications. Clinical implant dentistry and related research, 18(1), $204-212$.

Albuquerque, A. F. M., Cardoso, I. M. L., Silva, J. S. P. D., Germano, A. R., Dantas, W. R. M., \& Gondim, A. L. M. F. (2014). Levantamento de seio maxilar utilizando osso liofilizado associado a instalação imediata de implante do tipo cone morse: relato de caso.

Bacelar, S. M. D. A., \& Guimarães Neto, U. G. (2019). Sinus lift: Realização e técnicas cirúrgicas. Brazilian Journal of Implantology and Health Sciences, $1(5), 119-146$.

Batista, P. S., Junior, A. F. D. R., \& Wichnieski, C. (2011). Contribuição para o estudo do seio maxilar.Revista Portuguesa de Estomatología, Medicina Dentária e Cirugia Maxilofacial, 52(4), 235-239.

Batista, S. G., de Faria, M. R. S., Bochnia, J., de Agostinho Neto, O., de Mello, E. B., \& Magalhães, C. B. (2020). Levantamento de seio maxilar bilateral por duas técnicas diferentes com concomitante instalação de implantes: relato de caso. Revista Eletrônica Acervo Odontológico, 2, e5878-e5878.

Correia, J. M. M. D. V. (2020). Elevação do seio maxilar em medicina dentária: o estado da arte (Doctoral dissertation).

Correia, F., Almeida, R. F., Costa, A. L., Carvalho, J., \& Felino, A. (2012). Levantamento do seio maxilar pela técnica da janela lateral: tipos enxertos. Revista Portuguesa de Estomatologia, Medicina Dentária e Cirurgia Maxilofacial, 53(3), 190-196.

David, G. M., Vermudt, A., Ghizoni, J. S., Pereira, J. R., \& Pamato, S. (2018). Levantamento de seio maxilar: uma comparação de técnicas. Journal of Research in Dentistry, 6(2), 43-48.

Diniz, A. G., Sanches, H. R., Noro, G. A., Diniz, T. N. G., Neto, H. S., \& Junior, R. B. D. B. (2012). Estudo retrospectivo das cirurgias de elevação de seio maxilar. Revista da Associacao Paulista de Cirurgioes Dentistas, 66(1), 57-63.

Erdem, N. F., Çiftçi, A., \& Acar, A. H. (2016). Three-year clinical and radiographic implant follow-up in sinus-lifted maxilla with lateral window technique. Implant dentistry, 25(2), 214-221.

Esposito, M., Grusovin, M. G., Rees, J., Karasoulos, D., Felice, P., Alissa, R., \& Coulthard, P. (2010). Effectiveness of sinus lift procedures for dental implant rehabilitation: a Cochrane systematic. Eur J Oral Implantol, 3(1), 7-26.

Gandhi, Y. (2017). Sinus grafts: science and techniques - then and now. Journal of maxillofacial and oral surgery, 16(2), 135-144.

Guerrero, J. S. (2015). Lateral window sinus augmentation: Complications and outcomes of 101 consecutive procedures. Implant dentistry, 24(3), 354-361. 
Research, Society and Development, v. 10, n. 11, e238101119547, 2021

(CC BY 4.0) | ISSN 2525-3409 | DOI: http://dx.doi.org/10.33448/rsd-v10i11.19547

Kim, H. J., Yea, S., Kim, K. H., Lee, Y. M., Ku, Y., Rhyu, I. C., \& Seol, Y. J. (2020). A retrospective study of implants placed following 1-stage or 2-stage maxillary sinus floor augmentation by the lateral window technique performed on residual bone of $<4 \mathrm{~mm}$ : Results up to 10 years of follow-up. Journal of periodontology, 91(2), 183-193.

Lee, H. W., Lin, W. S., \& Morton, D. (2013). A retrospective study of complications associated with 100 consecutive maxillary sinus augmentations via the lateral window approach. International Journal of Oral \& Maxillofacial Implants, 28(3).

Lin, Y. H., Yang, Y. C., Wen, S. C., \& Wang, H. L. (2016). The influence of sinus membrane thickness upon membrane perforation during lateral window sinus augmentation. Clinical oral implants research, 27(5), 612-617.

Mazaro, J. V. Q., Pellizzer, E. P., Santiago Junior, J. F., Verri, F. R., \& Melo, C. C. D. (2013). Avaliação longitudinal de duas técnicas de sinus lift. Revista de Cirurgia e Traumatologia Buco-maxilo-facial,13(3), 09-16.

Misch, C. (2009). Implantes dentais contemporâneos. Elsevier Brasil.

Moraes, A. V., Tomasi B. A., \& Jonas L. O. (2018) Levantamento de seio maxilar: técnica lateral. Brazilian Journal of Focus in Scienctiae

Poli, G. H. D. S. (2017) Levantamento de seio maxilar pela técnica da janela lateral: relato de caso clínico. Monografia Especialização, Faculdade de Tecnologia de Sete Lagoas, Sete LAGOAS, Minas Gerais, Braisl.

Raja, S. V. (2009). Management of the posterior maxilla with sinus lift: review of techniques.Journal of Oral and Maxillofacial Surgery, 67(8), $1730-1734$.

Reis, J. C., \& Calixto, R. F. E. (2013). Cirurgia de levantamento de seio maxilar viabilizando o uso de implantes. INVESTIGAÇÃO, 13(3).

Salmen, F. S., Oliveira, M. R., Gabrielli, M. A. C., Piveta, A. C. G., Pereira Filho, V. A., \& Gabrielli, M. F. R. (2017). Enxerto ósseo para reconstrução óssea alveolar. Revisão de 166 casos. Revista do Colégio Brasileiro de Cirurgiões, 44(1), 33-40.

Testori, T., Scaini, R., Deflorian, M., \& Wallace, S. S. (2020). Sinus Augmentation: The Lateral Approach. Bone Augmentation by Anatomical Region: Techniques and Decision-Making, 283-308.

Tükel, H. C., \& Tatli, U. (2018). Risk factors and clinical outcomes of sinus membrane perforation during lateral window sinus lifting: analysis of 120 patients. International journal of oral and maxillofacial surgery, 47(9), 1189-1194.

Wallace, S. S., Tarnow, D. P., Froum, S. J., Cho, S. C., Zadeh, H. H., Stoupel, J., \& Testori, T. (2012). Maxillary sinus elevation by lateral window approach: evolution of technology and technique. Journal of Evidence Based Dental Practice, 12(3), 161-171. 